\title{
ESTUDO DA SECAGEM DA CANA-ENERGIA MEDIANTE MODELOS CINÉTICOS E PARÂMETROS DA LEI DE ARRHENIUS
}

\author{
M.G. SOUZA ${ }^{1}$, L. M. GUIFFO ${ }^{1}$, K. TANNOUS ${ }^{1}$, C.G. SANCHEZ ${ }^{2}$, P. MAZZAFERA ${ }^{3}$ \\ ${ }^{1}$ Universidade Estadual de Campinas, Faculdade de Engenharia Química \\ ${ }^{2}$ Universidade Estadual de Campinas, Faculdade de Engenharia Mecânica \\ ${ }^{3}$ Universidade Estadual de Campinas, Instituto de Biologia \\ E-mail para contato: melgesteira@gmail.com; katia@feq.unicamp.br
}

\begin{abstract}
RESUMO - $\mathrm{O}$ estudo da cinética de secagem de biomassas destinadas à produção de energia é de grande importância para a viabilização econômica e técnica de processos de termoconversão. Desta forma, foi conduzida a secagem isotérmica da Cana-Energia Saccharum spontaneum em estufa para três temperaturas: $60^{\circ} \mathrm{C}, 90^{\circ} \mathrm{C}$ e $103{ }^{\circ} \mathrm{C}$. Os dados experimentais obtidos foram comparados com sete modelos semi-teóricos, sendo estes: Lewis, Logarítmico, Page, Henderson e Pabis, Dois Termos, Exponencial de Dois Termos e Aproximação da Difusão. Os modelos de dois termos exponenciais apresentaram os melhores ajustes, com coeficientes de correlação superiores a 0,998. Baseando-se na Lei de Arrhenius foram determinados a energia de ativação e o fator pré-exponencial correspondentes às faixas de 21,8 - 27,6 kJ.mol-1 e 1,019-4,216 s $\mathrm{s}^{-1}$, respectivamente.
\end{abstract}

\section{INTRODUÇÃO}

Os desafios deste século com relação à diminuição da emissão de gases do efeito estufa e a busca por meios energéticos mais baratos, seguros e eficientes, fazem com que temas relacionados a fontes alternativas de energia ganhem destaque entre pesquisadores e lideranças políticas. Neste contexto, as biomassas são uma opção interessante e promissora. No entanto, o baixo poder calorífico devido ao alto teor de umidade desse material limita sua aplicação como combustível alternativo, tornando a secagem fundamental para sua viabilização técnica e econômica.

No Brasil estão presentes várias biomassas que condizem com a ampla biodiversidade do país. No entanto, as plantações de cana-de-açúcar e a enorme quantidade produzida deste material chamam a atenção para esta biomassa. Recentemente, uma nova variedade de canade-açúcar, a Cana-Energia, vem ganhando espaço no agronegócio e no meio científico por ser mais produtiva e mais resistente que a cana convencional (Kim e Day, 2011).

Tendo em vista essa nova ótica a respeito da geração de energia, este trabalho tem por objetivo estudar a cinética de secagem em estufa de uma espécie de Cana-Energia (Saccharum spontaneum), a diferentes temperaturas, utilizando modelos semi-teóricos, bem como a determinação dos parâmetros da Lei de Arrhenius (Energia de ativação, fator préexponencial). 


\section{MATERIAIS E MÉTODOS}

\subsection{Procedimento Experimental de Secagem}

A Cana-Energia do tipo Saccharum spontaneum (tempo de plantio de 18 meses) foi coletada no IB/Unicamp. O caule foi cortado em pedaços cilíndricos entre 4 e $6 \mathrm{~cm}$ de comprimento e alocados em placas de alumínio de $12,5 \mathrm{~cm}$ de diâmetro de modo a formar uma camada única de biomassa. Os ensaios foram conduzidos em estufa Q314M242 (Fabricante Quimis, Diadema, Brasil). As cinéticas de secagem foram feitas para $60^{\circ} \mathrm{C}, 90^{\circ} \mathrm{C}$ e $103{ }^{\circ} \mathrm{C}$. Não foram consideradas temperaturas maiores para evitar volatilização de componentes. A amostra foi colocada na estufa permanencendo por incrementos de cinco minutos, sendo removida e alocada em um dessecador de vidro pelo mesmo período de tempo para que esfriasse. $\mathrm{O}$ sistema foi então pesado. Esse processo foi realizado até o equilibrio da massa da amostra. Os ensaios foram feitos em duplicata e para a análise final foi feita a média dos resultados obtidos em cada temperatura.

\subsection{Modelos Cinéticos Semi-Teóricos e Ajustes}

Primeiramente, os dados experimentais foram normalizados aplicando a Equação (1), proposta por Lewis (1921),

$$
M R=\frac{m-m_{e}}{m_{0}-m_{e}}
$$

na qual $M R$ representa a razão de umidade adimensional, $m$ a massa da amostra em um instante de tempo, $m_{e}$ a massa da amostra no equilíbrio e $m_{\mathrm{o}}$ a massa inicial da amostra. Após, os dados experimentais foram validados aplicando modelos semi-teóricos (Tabela 1).

Tabela 1 - Modelos Cinéticos Semi-Teóricos de Secagem

\begin{tabular}{cccc}
\hline Modelo & Equação & Parâmetros & Autor \\
\hline Lewis & $M R=\exp (-k t)$ & $\mathrm{k}$ & Lewis (1921) \\
\hline Page & $M R=\exp \left(-k t^{n}\right)$ & $\mathrm{k}, \mathrm{n}$ & Page (1949) \\
\hline Henderson e Pabis & $M R=a \exp (-k t)$ & $\mathrm{k}, \mathrm{a}$ & $\begin{array}{c}\text { Henderson e } \\
\text { Pabis (1961) }\end{array}$ \\
\hline Aproximação da difusão & $M R=a \exp (-k t)+(1-a) \exp (-k b t)$ & $\mathrm{k}, \mathrm{a}, \mathrm{b}$ & $\begin{array}{c}\text { Sharaf-Eldeen } \\
(1980)\end{array}$ \\
\hline Modelo de dois termos & $M R=a \exp \left(-k_{0} t\right)+c \exp \left(-k_{b} t\right)$ & $\mathrm{k}_{0}, \mathrm{k}_{\mathrm{b}}, \mathrm{a}, \mathrm{c}$ & $\begin{array}{c}\text { Noomhorm e } \\
\text { Verma (1986) }\end{array}$ \\
\hline Logarítmico & $M R=a \exp (-k t)+c$ & $\mathrm{k}, \mathrm{a}, \mathrm{c}$ & $\begin{array}{c}\text { Akgun e Doymaz } \\
(2005)\end{array}$ \\
\hline $\begin{array}{c}\text { Modelo exponencial de } \\
\text { dois termos }\end{array}$ & $M R=a \exp (-k t)+(1-a) \exp (-k a t)$ & $\mathrm{k}$ & Doymaz (2011) \\
\hline
\end{tabular}

O modelo de Lewis prediz o comportamento aproximado da cinética de secagem somente para uma camada fina de amostra e umidade de equilíbrio desprezível. O parâmetro empírico $k$, foi denominado como a "constante de secagem". Tendo Lewis (1921) como base, 
os modelos de Page, Henderson \& Pabis e Logarítmico foram propostos acrescentando coeficientes empíricos para ajuste matemático (Senadeera et al., 2003; Sharaf-Eldeen, 1980). Os modelos de dois termos (aproximação da difusão, modelo de dois termos, e modelo exponencial de dois termos) por sua vez, foram baseados no truncamento no segundo termo da série que representa a solução do modelo difusional ( $2^{\circ}$ Lei de Fick).

Os parâmetros dos respectivos modelos foram encontrados pela ferramenta Solver do software Excel, através do método Gradiente Reduzido Generalizado (GRG). O método dos mínimos quadrados foi utilizado, no qual se assume que a soma do quadrado das diferenças entre MR teóricos e experimentais é igual a zero, conforme Equação 2.

$$
\sum_{i=1}^{n}\left(M R_{\text {teórico }}-M R_{\text {exp erimental }}\right)_{i}^{2}=0
$$

Para medir a acurácia dos dados teóricos e experimentais foi calculado o coeficiente de correlação $(r)$ mediante a Equação (3). Este representa o grau de relação existente entre duas variáveis. Para complementar as informações que fornece o $r$, foi calculado o erro quadrático médio (RMSE) utilizando a Equação (4), que quantifica a extensão da variação dos dados calculados com os modelos teóricos em relação aos dados experimentais.

$$
\begin{aligned}
& r=\frac{\sum(x-\bar{x})(y-\bar{y})}{\sqrt{\sum(x-\bar{x})^{2} \sum(y-\bar{y})^{2}}} \\
& R M S E=\sqrt{\frac{\sum(x-y)^{2}}{n}}
\end{aligned}
$$

Sendo $\mathrm{x} \mathrm{e}^{\bar{x}} \mathrm{o}$ valor experimental e valor médio experimental da umidade normatizada MR, respectivamente; y e $\bar{y}$ o valor teórico e o valor teórico de MR médio calculado para cada modelo cinético, respectivamente.

\subsection{Energia de Ativação e Fator Pré-Exponencial de Arrhenius}

A Lei de Arrhenius (Equação 5) foi utilizada para descrever diversos modelos cinéticos uma vez que relaciona a constante da taxa de reação $k$ com a temperatura $T$. Apesar de ser definida somente para reações em fase gasosa, esta lei apresenta um bom ajuste para dados cinéticos experimentais de secagem (White et al., 2011),

$$
k(T)=A \exp \left(\frac{-E}{R T}\right)
$$

na qual, $k$ é a constante da taxa de Arrhenius em s ${ }^{-1}, A$ é o fator pré-exponencial em s ${ }^{-1}, E$ é a energia de ativação em $\mathrm{kJ}^{-\mathrm{mol}^{-1}}, T$ é a temperatura em $\mathrm{K}$ e $R$ é a constante universal dos gases em kJ.mol${ }^{-1} \mathrm{~K}^{-1}$.

Nos modelos de um termo exponencial (Lewis, Page, Logarítmico e Henderson \& Pabis) a constante da taxa de secagem $(k)$ é dada diretamente através dos parámetros provenientes dos ajustes descritos no item 2.2. Desta forma, para obter a energia de ativação e 
o fator pré-exponencial, basta realizar um ajuste linear em termos de $\ln (k)$ e $1 / T$ (Senandeera et al., 2003; Liu et al., 2013).

\section{RESULTADOS E DISCUSSÃO}

\subsection{Curvas de Secagem}

Dados experimentais da umidade normatizada (MR) em função do tempo foram obtidos para as três temperaturas $\left(60^{\circ} \mathrm{C}, 90^{\circ} \mathrm{C}\right.$ e $\left.103^{\circ} \mathrm{C}\right)$. Na Figura 1 , pode-se observar que a temperatura possui uma influência no tempo de secagem obtido pelo sólido, isto é, à medida que a temperatura de secagem aumenta, o tempo diminui.

Figura 1 - Variação de umidade em relação ao tempo da cana-de-açúcar Saccharum spontaneum a diferentes temperaturas

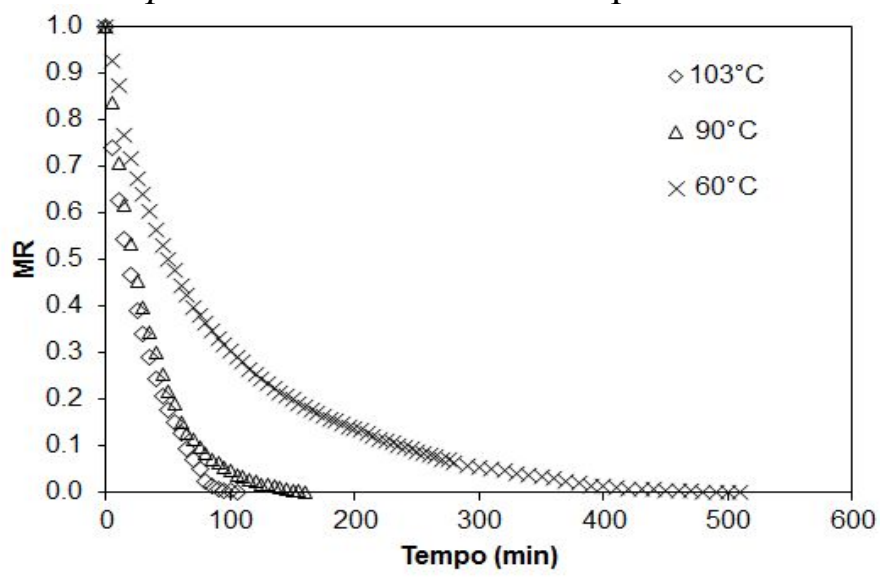

Embora o tempo de equilíbrio decresça em relação ao aumento da temperatura (Figura 1), a diferença entre a curva de secagem entre as temperaturas de $90^{\circ} \mathrm{C}$ e de $103^{\circ} \mathrm{C}$ é muito pequena, refletindo em um tempo de equilíbrio muito próximo. Este fato pode implicar na otimização de um possível sistema de secagem já que a temperatura de operação e, consequentemente, a energia gasta para a sua operação podem ser diminuídas sem a alteração significativa do tempo de secagem.

\subsection{Aplicação dos modelos cinéticos semi-teóricos}

As Figuras 2(a) e 2(b) exemplificam as curvas de secagem da Cana-Energia obtidas experimentalmente e através de dados semi-teóricos para a temperatura de $103^{\circ} \mathrm{C}$. A CanaEnergia, por ser um material poroso e higroscópico, contém água nas cavidades e nas paredes das fibras, prevalecendo o transporte difusivo. Com isso, as curvas foram divididas em duas etapas associadas a perda de água presente na amostra: etapa (1) ocorre a difusão da água ligada às paredes da fibra por pontes de hidrogênio; etapa (2) aumento da resistência interna à secagem, implicando em uma diminuição da taxa de perda de massa $(\Delta \mathrm{MR} / \Delta \mathrm{t})$ até atingir a umidade de equilíbrio (Jankowsky e Santos, 2004). Para a primeira etapa a taxa de perda de massa foi de $3,4 \times 10^{-4} \mathrm{~s}^{-1}$, correspondente a $66 \%$ de perda, enquanto que na segunda etapa a taxa diminuiu para $4,2 \times 10^{-5} \mathrm{~s}^{-1}$, equivalente a $34 \%$.

Com relação aos modelos também apresentados na Figura 2, observa-se que os modelos 
de um termo apresentaram coeficiente de correlação $(r)$ entre 0,9903 e 0,9995 , e erro

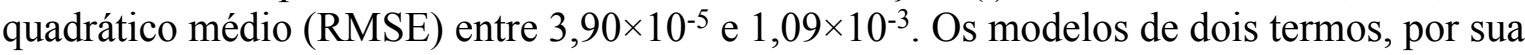

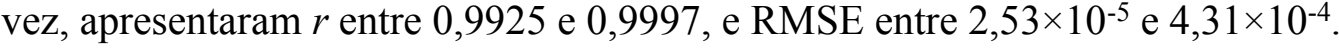

Figura 2 - Secagem da Cana-Energia Saccharum spontaneum a $103^{\circ} \mathrm{C}$ - (a) Modelos de um termo exponencial (b) Modelos de dois termos exponenciais
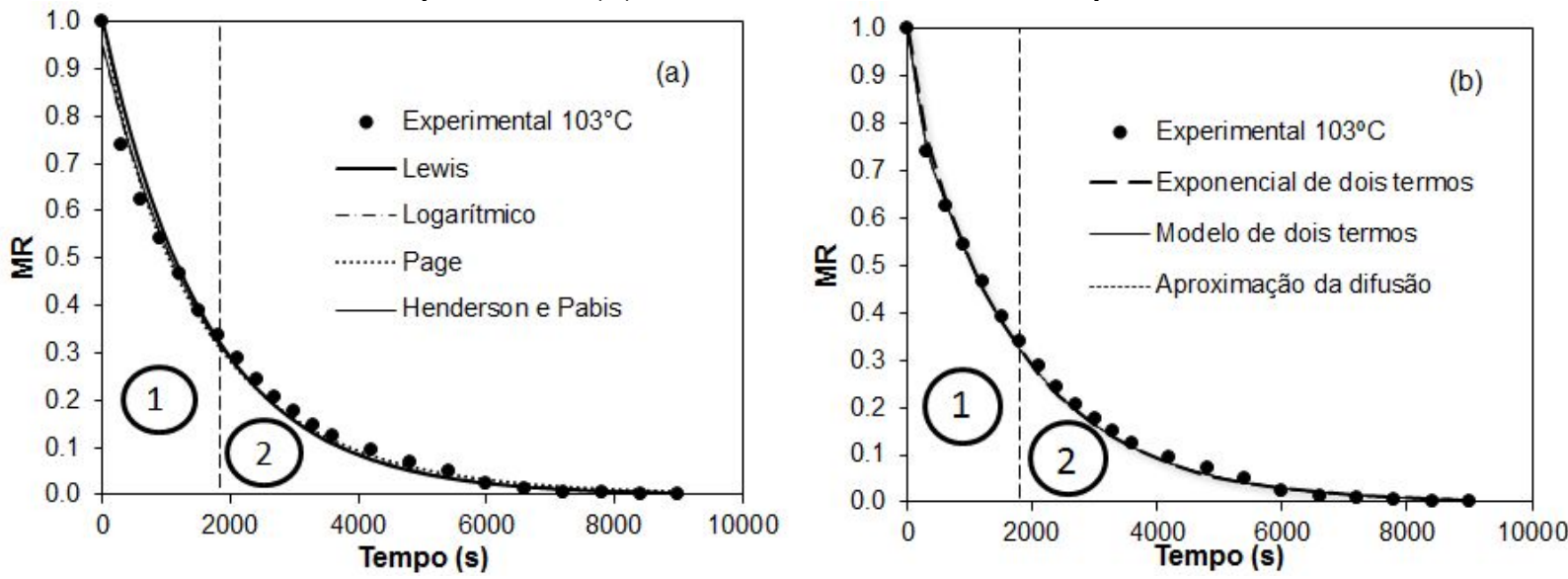

A secagem da Saccharum spontaneum possui uma transferência de massa nas coordenadas radial e axial, predominando a difusividade da água. Apesar dos modelos mais simples representarem satisfatoriamente os dados experimentais, estes foram baseados na lei de resfriamento de Newton, considerando um sistema de camada fina. Portanto, os modelos semi-teóricos de dois termos representam melhor fisicamente os resultados, pois são soluções da segunda lei de Fick, considerando um coeficiente de difusão constante e assumindo geometria esférica. A acurácia dos modelos de dois termos não foi comprometida apesar do sistema estudado possuir uma geometria cilíndrica.

\subsection{Energia de Ativação e Fator Pré-Exponencial de Arrhenius}

Através dos dados experimentais foi possível obter os parâmetros de Arrhenius $(E, A)$ que descrevem a secagem da cana Saccharum spontaneum. A $E$ representa a energia mínima a ser fornecida ao sistema para que se inicie a eliminação da água presente no material. As energias variaram entre $21,8 \mathrm{~kJ} . \mathrm{mol}^{-1}$ e $27,6 \mathrm{~kJ} \cdot \mathrm{mol}^{-1}$. O $A$, correspondente à frequência de ocorrência da secagem, variou entre $1,019 \mathrm{~s}^{-1}$ e $4,216 \mathrm{~s}^{-1}$. Na literatura foram encontrados alguns exemplos de $E$ de secagem, a saber: 19,82 kJ/mol para resíduos vegetais (Lopez et al., 2000), 17,97 kJ/mol para resíduo sólido de azeitona (Akgun e Doymaz, 2005) e 39,47 kJ/mol para feijão (Senadeera et al., 2003). Um comparativo entre modelos semi-teóricos foi realizado (Tabela 3), sendo o Logarítmico mais próximo aos dados experimentais.

Tabela 3 - Fator pré-exponencial (A) e energia de ativação (E) correspondente a cada modelo de um termo exponencial da cinética de secagem

\begin{tabular}{cccc}
\hline Modelo & $\mathbf{A ~ ( \mathbf { s } ^ { \mathbf { 1 } } )}$ & $\mathbf{E}\left(\mathbf{k J} . \mathbf{~ m o l}^{\mathbf{1}}\right)$ & $\boldsymbol{r}$ \\
\hline Lewis & 3,475 & 26,871 & 0,964 \\
Logarítmico & 4,010 & 27,483 & 0,992 \\
Page & 1,019 & 21,836 & 0,928 \\
Henderson \& Pabis & 4,216 & 27,630 & 0,987 \\
\hline
\end{tabular}




\section{CONCLUSÃO}

Os dados experimentais e semi-teóricos obtidos e os fatos explicitados permitiram concluir que na secagem da Cana-Energia as temperaturas de $90{ }^{\circ} \mathrm{C}$ e $103^{\circ} \mathrm{C}$ apresentaram tempos de equilíbrio muito próximos. Os modelos cinéticos aplicados descreveram adequadamente a secagem comprovados através dos $r$ entre 0,9903 e 0,9995 para os modelos de um termo e entre 0,9925 e 0,9997 para os modelos de dois termos. No entanto, destacamse os modelos de dois termos baseados na teoria difusional, condizente à secagem estudada. A obtenção dos parâmetros de Arrhenius revela que é necessária uma energia de ativação entre

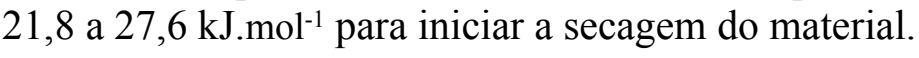

\section{AGRADECIMENTOS}

Os autores agradecem ao CNPq e CAPES pelo apoio financeiro, bem como ao Prof. Dr. Antonio C. L. Lisboa (FEQ/Unicamp) pelo apoio técnico.

\section{REFERÊNCIAS}

AKGUN, N. A.; DOYMAZ, I. Modelling of olive cake thin-layer drying process. Journal of Food Engineering, n. 68, v. 4, p. 55-461, 2005.

DOYMAZ, I. Drying of green bean and okra under solar energy. Chemical Industry and Chemical Engineering Quarterly, v. 17, p. 199-205, 2011.

HENDERSON, S.; PABIS, S. Grain drying theory. II - Temperature effects on drying coefficients. Journal of Agricultural Engineering Research, v. 6, p. 169-74, 1961.

JANKOWSKY, I. P.; SANTOS, G.R. V. Drying behavior and permeability of Eucalyptus grandis Lumber. In: Proceedings of the 14th International Drying Symposium, v. B, p. 1385-1389, 2004.

KIM, M.; DAY, D.F. Composition of sugar cane, energy cane, and sweet sorghum suitable for ethanol production at Louisiana sugar mills. Journal of Industrial Microbiology \& Biotechnology, p. 803-807, 2011.

LEWIS, W. The rate of drying of solids materials. Journal of Industrial and Engineering Chemistry, v. 13, p. 427-432, 1921.

LIU, H.M.; CHEN M.Q., HAN, Z.L.; FU, B.A.; Isothermal kinetics based on two-periods scheme for co-drying of biomass and lignite. Thermochimica Acta 573, p. 25-31, 2013.

LOPEZ, A.; IGUAZ, A.; ESNAZ, A.; VIRSEDA, P. Thin-layer drying behavior of vegetable wastes from wholesale market. Drying Technology, v.18, p. 995-1006.

NOOMHORM, A.; VERMA, L. Generalized single-layer rice drying models. Transactions of the ASAE, v. 29(2), p. 587-591, 1986.

PAGE, G. E. Factors influencing the maximum rates of air drying shelled corn in thin layers. Dissertação (Mestrado), Lafayette: Purdue University, 1949.

SENADEERA, W.; BHANDARI, B. R.; YOUNG, G.; WIJESINGHE, B. Influence of shapes of selected vegetable materials on drying kinetics during fluidized bed drying. Journal of Food Engineering, v. 58, p. 277-283, 2003.

WHITE, J. E.; CATALLOB, W. J.; LEGENDREA, B. L. Biomass pyrolysis kinetics: A comparative critical review with relevant agricultural residue case studies. Journal of Analytical and Applied Pyrolysis, v. 91, p. 1-33, 2011.

SHARAF-ELDEEN, Y.I.;. BLAISDELL, J. L; HAMDY, M. Y. A model for ear corn drying. Transactions of ASAE, v. 23(5), p.1261-1265, 1980. 